Original Research Paper

\title{
Study the Relationship between the Microstructure and Characteristics of Quinoa Starch by Compared with Common Cereal Starches
}

\author{
${ }^{1}$ Menyue Jiao, ${ }^{2}$ Yumei Gao and ${ }^{1}$ Yiling Tian \\ ${ }^{1}$ College of Food Science and Technology, Hebei Agricultural University, China \\ ${ }^{2}$ Life Science and Food Engineering, Hebei University of Engineering, China
}

\author{
Article history \\ Received: 05-09-2020 \\ Revised: 14-12-2020 \\ Accepted: $15-12-2020$ \\ Corresponding Author: \\ Yiling Tian \\ College of Food Science and \\ Technology, Hebei Agricultural \\ University, China \\ Email: tougaotian@126.com
}

\begin{abstract}
The amylose and amylopectin contents, viscoelastic properties, freeze-thaw stability and Scanning Electron Micrographs (SEM) of quinoa (Chenopodium quinoa Willd) were studied to explore the microstructural property relationships. Compared with wheat, corn, rice and waxy rice starches, quinoa starch had special characteristics connected to its unique microstructure. The high ratio of amylopectin to amylose (12.03) in quinoa starch affected its functional and nutritional properties. Quinoa starch had the lowest setback (97 BU) showed that it had the best anti-aging ability of those cereal starches studied (153.5-1068 BU). The poor freeze-thaw stability of quinoa starch and the structure of its paste are related to its amylopectin contain extremely numerous Longest Chain (LC) and short chains, a few long chains. By comparing its size and shape with those of other types of starch granule, it was found that the small uniform size $(1 \mu \mathrm{m})$ and polygonal structure of the quinoa starch granule affect its susceptibility to enzymes.
\end{abstract}

Keywords: Quinoa Starch, Microstructure, Physicochemical Properties, Anti-Aging Ability

\section{Introduction}

Quinoa, a cereal crop native to South America, has been cultivated for centuries. In view of its significant role in providing safe and nutritious food and eliminating poverty and with starch being the main component of quinoa seed (55\%), the relationship between the microstructure and properties of quinoa starch needs further study.

Starch is mainly constituted of amylose and amylopectin. The structure of amylopectin is closely related to the physical properties of starch such as gelatinization (Bertoft et al., 2008). The ratio of amylose to amylopectin is also important to both the functionality and the nutritional properties of starch and starch-based products (Kaufman et al., 2015). Quinoa starch is composed of very small granules with unique properties but its molecular structure remains largely unknown. At present, many studies have investigated the properties of the ultrafine particles of normal cereal starches such as starch content, gelatinization, crystal structure and morphology, to reveal the relationship between the structural composition of starch and its inherent nature and function (Wang et al., 2011).
Starch is often incorporated into many frozen foods as one of the main raw materials or additives (Sae-Kang and Suphantharika, 2006). The concept of freeze-thaw stability is commonly used to measure the ability of starch to withstand negative physical changes during freezing and thawing (Liu et al., 2012). The sensory quality and the shelf life of starchy food would be adversely affected by the loss of water during these FTC (Teng et al., 2013). Changes in viscosity can also influence other characteristics of starch, such as their pasting properties, thermal stability and their susceptibility to retrogradation.

The present study aims to study the relationship between the unique properties of quinoa starch and its microstructure, which will help to provide a better understanding of this starch, to explore further applications and to see whether this relationship could be applied to other types of starch. Quinoa is a crop with high nutritional value, but currently its processing methods are limited to boiling and salad making and the degree of deep processing and commercialization is not high. The structural properties obtained in this study can provide references for more food applications, such as quick-frozen foods, pre-gelatinized foods and fitness 
meal recipes and accelerate their commercialization. In addition, this article compares common starches and based on their similarities in characteristics, quinoa can be used as a substitute to enrich the variety and increase its commercial value.

\section{Materials and Methods}

\section{Materials}

All chemicals used were reagent grade. The potato Amylose (A0512) and potato Amylopectin (A8515) were bought from Sigma-Aldrich (St. Louis, MO, USA).

\section{Extraction of Starch}

At present, there is no marketed quinoa starch and the purity of starches on the market is much different, which could not meet the requirements of comparison. Extracted flour repeatedly with $0.25 \% \mathrm{NaOH}$ in $1: 5$ ratio till free from protein. Centrifuged at $1620 \times \mathrm{g}$ for $15 \mathrm{~min}$ and then washed thoroughly till free of $\mathrm{NaOH}$. Passed them through 200 mesh filter cloth, dry at $50^{\circ} \mathrm{C}$, sieve them through 60 mesh and store (Ahamed et al., 1996; Kusumah et al., 2020).

\section{Amylose and Amylopectin Content}

The amylose and amylopectin contents of the isolated starches were analyzed using the dual-wavelength method. Ten $\mathrm{ml}$ of $0.5 \mathrm{~mol} / \mathrm{L} \mathrm{KOH}$ was added to $100 \mathrm{mg}$ of the starch sample (based on dry weight) then the suspension was heated to $60^{\circ} \mathrm{C}$ for $20 \mathrm{~min}$. The dispersed sample was then transferred to a $50 \mathrm{~mL}$ volumetric flask as the standard working starch solution.

Two $\mathrm{mL}$ of the amylose or amylopectin standard solution were placed in a $100 \mathrm{~mL}$ beaker, distilled water was added to make up to $25 \mathrm{~mL}, 0.1 \mathrm{~mol} / \mathrm{L} \mathrm{HCl}$ solution was added to adjust the $\mathrm{pH}$ value to 3.5 , then the solution was transferred to a $50-\mathrm{mL}$ volumetric flask. After adding $0.5 \mathrm{~mL}$ iodine reagent, distilled water was added to make up to $50 \mathrm{~mL}$. After standing at $20^{\circ} \mathrm{C}$ for $30 \mathrm{~min}$, the sample was placed in a UV spectrophotometer (UV1700PC, Macylab Instrument Inc, Shanghai, China) for a full wavelength scan to determine the measurement wavelength and reference wavelength using the dual wavelength absorbance point mapping method and the iodine reagent as the blank (Kaufman et al., 2015).

\section{Measuring the Viscoelastic Properties}

The viscoelastic properties were measured as described by (Jacobs et al., 1995) using a visco-amylograph (Brabender $\mathrm{GmbH}$ and Co. KG, Duisburg, Germany) equipped with a $500 \mathrm{~cm}^{*} \mathrm{~g}$ cartridge. The temperature-time conditions for the starch suspensions (6\%, w/w; $500 \mathrm{~g}$ total weight) were: A heating step from 35 to $95^{\circ} \mathrm{C}$ at $1.5^{\circ} \mathrm{C} / \mathrm{min}$; a holding phase at $95^{\circ} \mathrm{C}$ for 20 min; a cooling step from 95 to $35^{\circ} \mathrm{C}$ at $1.5^{\circ} \mathrm{C} / \mathrm{min}$; then a holding phase at $35^{\circ} \mathrm{C}$ for $20 \mathrm{~min}$. The Pasting Temperature (PT), Peak Viscosity (PV), Hot Paste Viscosity (HPV), Cool Paste Viscosity (CPV) and the derivative parameters, Breakdown $(\mathrm{BD}=\mathrm{PV}-\mathrm{HPV})$ and Setback (SB = CPV-HPV) were recorded with the viscosity parameters presented in BU.

\section{Freeze-Thaw Stability of Starch}

The freeze-thaw stability was measured using the methods of (Ahamed et al., 1996; Yan et al., 2010). The freeze-thaw stability of starch was studied by repeatedly freezing and thawing 5\% starch pastes, then measuring the amount of water separated after centrifuging the thawed paste. Starch milk with a mass fraction of $5 \%$ was prepared and heated at a temperature of $95^{\circ} \mathrm{C}$, stirred until the volume of starch paste was unchanged then cooled at room temperature. The starch pastes were frozen at $-10^{\circ} \mathrm{C}$ for $18 \mathrm{~h}$ then thawed at room temperature for $6 \mathrm{~h}$. The starch suspensions were then centrifuged at $1620 \times \mathrm{g}$ for $10 \mathrm{~min}$. The supernatant obtained was carefully removed and the swollen starch Sediment was weighed (SS). The analyses were performed in triplicate. The freeze-thaw stability was calculated as follows:

Water splitting rate $(g / g)=(S W-S S) / S W \times 100 \%$

Where:

$S W=$ Weight of wet sediment

$S S=$ Weight of swollen starch sediment

\section{Scanning Electron Microscopy}

The morphology of the starch granules was studied using an XL-3 environmental scanning electron microscope (Philips, Eindhoven and The Netherlands). The dried samples were mounted on a metal stub, coated with gold powder to make the sample conductive, then the images were obtained at an accelerating potential of $15 \mathrm{kV}$ under low vacuum.

\section{Statistical Analysis}

The means, standard deviations and coefficients of variation were calculated using an excel spreadsheet (Microsoft Corp., Redmond, WA, USA).

\section{Results}

\section{Amylose and Amylopectin Content}

The amylose and amylopectin contents and the ratio of amylose to amylopectin determined in common cereal starches and quinoa starch are shown in Table 1. The purity of starches ranges from 91.24 to $99.96 \%$. The amylose content in quinoa starch was $7.00 \%$, generally comparable with previous studies which used the Con a 
precipitation-based method to measure the true amylose content, but reported higher amylose contents (8.22-9.30\%) (Steffolani et al., 2013; Linsberger-Martin et al., 2012). Watanabe et al. (2007) used Gel Permeation Chromatography (GPC) to measure amylose content and for quinoa starch reported values ranging from 5.2 to $10.9 \%$. The amylose content of the other cereal starches, except waxy rice starch, were 2.46 5.08 times higher than that of quinoa starch. The ratio of amylose to amylopectin in quinoa starch was 12.03, a much higher ratio than for the other starches.

\section{Measuring the Viscoelastic Properties}

When starch is heated continuously in excess water with stirring, the starch granules swell irreversibly, accompanied by the leaching of amylose and possibly the solubilization of amylopectin, resulting in the formation of a paste. To a certain extent, the starch pasting behavior determines its cooking quality and functionality, because starch is the main component of these grains. The pasting properties determined are shown in Table 2.

Table 2 shows that the swelling power of quinoa starch was similar to that of the corn and rice starches and its shear resistance at high temperature was similar to that of the waxy rice and corn starches. The hardness of the quinoa starch paste at room temperature was similar to that of the waxy rice and wheat starches. The values of PV, CPV and HPV for the quinoa starch were not high, but the thermal stability of its starch paste was very good. It was also the most difficult of all the starches to be aged.

The setback viscosity, SB, represents the tendency of a paste to retrograde and the amylose to reassociate: Quinoa starch had the lowest SB (97 BU). It is obvious that quinoa starch paste had the best antiaging properties of these starch pastes. This indicated that quinoa starch paste can still maintain a degree of softness and elasticity after cooling for a certain period after gelatinization.

Table 3 shows the freeze-thaw stability of the wheat, corn, rice and waxy rice and quinoa starches at 5\% level. (Ahamed et al., 1996) reported that quinoa starch has excellent freeze-thaw stability, as restated in many review articles (Tang et al., 2002; Navruz-Varli and Sanlier, 2016), but few studies have verified this.

The freeze-thaw stability of starch gels, measured as \% syneresis, was determined after nine FTCs (Table 3). Of the starches tested, the gels of wheat, corn, rice and quinoa lost their stability rapidly after the 1st FTC where the degrees of syneresis were more than $20 \%$. Subsequently, further FTC slightly increased the degrees of syneresis, reaching 32.73-64.36\% after the 3rd cycle. In the present study, waxy rice starch was the most stable gel because no syneresis was observed until after the 7th FTC.

Table 1: The amylose and amylopectin contents of common cereal starches and quinoa starch

\begin{tabular}{lllr}
\hline & Amylose content $(\%)$ & Amylopectin content $(\%)$ & Amylopectin content/Amylose content \\
\hline Wheat & $35.54 \pm 0.23$ & $62.17 \pm 0.32$ & 1.75 \\
Corn & $31.88 \pm 0.14$ & $60.27 \pm 0.55$ & 1.89 \\
Rice & $18.68 \pm 0.14$ & $81.28 \pm 0.30$ & 4.35 \\
Waxy rice & $0.65 \pm 0.045$ & $91.73 \pm 0.11$ & 141.12 \\
Quinoa & $7.00 \pm 0.29$ & $84.24 \pm 1.54$ & 12.03 \\
\hline
\end{tabular}

Values are means \pm SD $(n=3)$

Table 2: Brabender viscosity parameters of quinoa and other starches

\begin{tabular}{lllllll}
\hline & PV $(\mathrm{BU})$ & $\mathrm{CPV}(\mathrm{BU})$ & $\mathrm{HPV}(\mathrm{BU})$ & $\mathrm{BD}(\mathrm{BU})$ & $\mathrm{SB}(\mathrm{BU})$ & $\mathrm{PT}\left({ }^{\circ} \mathrm{C}\right)$ \\
\hline Wheat & $93.5 \pm 3.54$ & $235 \pm 7.07$ & $78.5 \pm 2.12$ & $15 \pm 1.41$ & $164.5 \pm 2.12$ & $88.35 \pm 0.21$ \\
Corn & $324 \pm 5.66$ & $669 \pm 0$ & $258 \pm 2.83$ & $66 \pm 2.83$ & $416.5 \pm 10.61$ & $82.55 \pm 0.071$ \\
Rice & $289 \pm 2.83$ & $829.5 \pm 23.33$ & $132.5 \pm 0.71$ & $156.5 \pm 2.12$ & $1068 \pm 25.46$ & $69.65 \pm 0.21$ \\
waxy rice & $779 \pm 1.41$ & $441 \pm 14.14$ & $297.5 \pm 2.12$ & $481.5 \pm 0.71$ & $153.5 \pm 3.53$ & $68.15 \pm 0.071$ \\
Quinoa & $319 \pm 4.24$ & $385 \pm 1.41$ & $293.5 \pm 3.54$ & $25.5 \pm 0.71$ & $97 \pm 2.83$ & $65 \pm 0.28$ \\
\hline
\end{tabular}

Values shown as mean \pm standard deviation $(\mathrm{n}=3)$; PT: Pasting Temperature; PV: Peak Viscosity; CPV: Cold Paste Viscosity; HPV: Hot Paste Viscosity; BD: Breakdown (PV-HPV); and SB: Setback (CPV-HPV)

Table 3: The extent of water separation (\%) of seven types of starch gel after nine freeze-thaw cycles

\begin{tabular}{|c|c|c|c|c|c|c|c|c|c|c|}
\hline & \multicolumn{10}{|c|}{ Water separation $/ \%$} \\
\hline & 1 & 2 & 3 & 4 & 5 & 6 & 7 & 8 & 9 & TW \\
\hline Wheat & $34.29 \pm 0.80$ & $6.48 \pm 1.75$ & $0.4 \pm 0.20$ & $0 \pm 0$ & $0 \pm 0$ & $2.10 \pm 1.21$ & $0.46 \pm 0.62$ & $0 \pm 0$ & $0 \pm 0$ & 43.72 \\
\hline Corn & $36.95 \pm 0.80$ & $11.30 \pm 4.16$ & $13.50 \pm 3.26$ & $1.04 \pm 0.22$ & $0.28 \pm 0.042$ & $0 \pm 0$ & $1.13 \pm 0.028$ & $0.18 \pm 0.11$ & $0 \pm 0$ & 64.36 \\
\hline Rice & $24.58 \pm 5.75$ & $4.33 \pm 4.26$ & $3.76 \pm 5.22$ & $1.38 \pm 1.94$ & $1.48 \pm 2.06$ & $0 \pm 0$ & $1.6 \pm 1.92$ & $0.11 \pm 0.15$ & $0 \pm 0$ & 37.22 \\
\hline Waxy rice & $0 \pm 0$ & $0.015 \pm 0.021$ & $0 \pm 0$ & $0 \pm 0$ & $0 \pm 0$ & $0 \pm 0$ & $24.88 \pm 35.16$ & $1.42 \pm 1.98$ & $0 \pm 0$ & 26.32 \\
\hline Quinoa & $45.57 \pm 1.35$ & $7.24 \pm 1.60$ & $0.72 \pm 0.82$ & $0.59 \pm 0.81$ & $0.04 \pm 0.021$ & $0 \pm 0$ & $0.01 \pm 0.014$ & $0.59 \pm 0.81$ & $0 \pm 0$ & 54.75 \\
\hline
\end{tabular}

TW: Total water separation (\%); Values shown as mean \pm standard deviation $(\mathrm{n}=3)$ 
Menyue Jiao et al. / American Journal of Biochemistry and Biotechnology 2020, 16 (4): 561.567 DOI: 10.3844/ajbbsp.2020.561.567

Table 4: The classification and microscopic characteristics of several edible starch granules

\begin{tabular}{llll}
\hline Starch & Large particle diameter $(\mu \mathrm{m})$ & small particle diameter $(\mu \mathrm{m})$ & Smooth degree \\
\hline Tuberose, flat & & & Smooth \\
Wheat & $25-32$ & $2-8$ & Smooth edges and corners \\
Polyhedral & $5-15$ & & Sharp edges and corners \\
Corn & $4-8$ & & Sharp edges and corners \\
Rice & $4-8$ & & Smooth edges and corners \\
Waxy rice & $1-1.5$ & & \\
Quinoa &
\end{tabular}
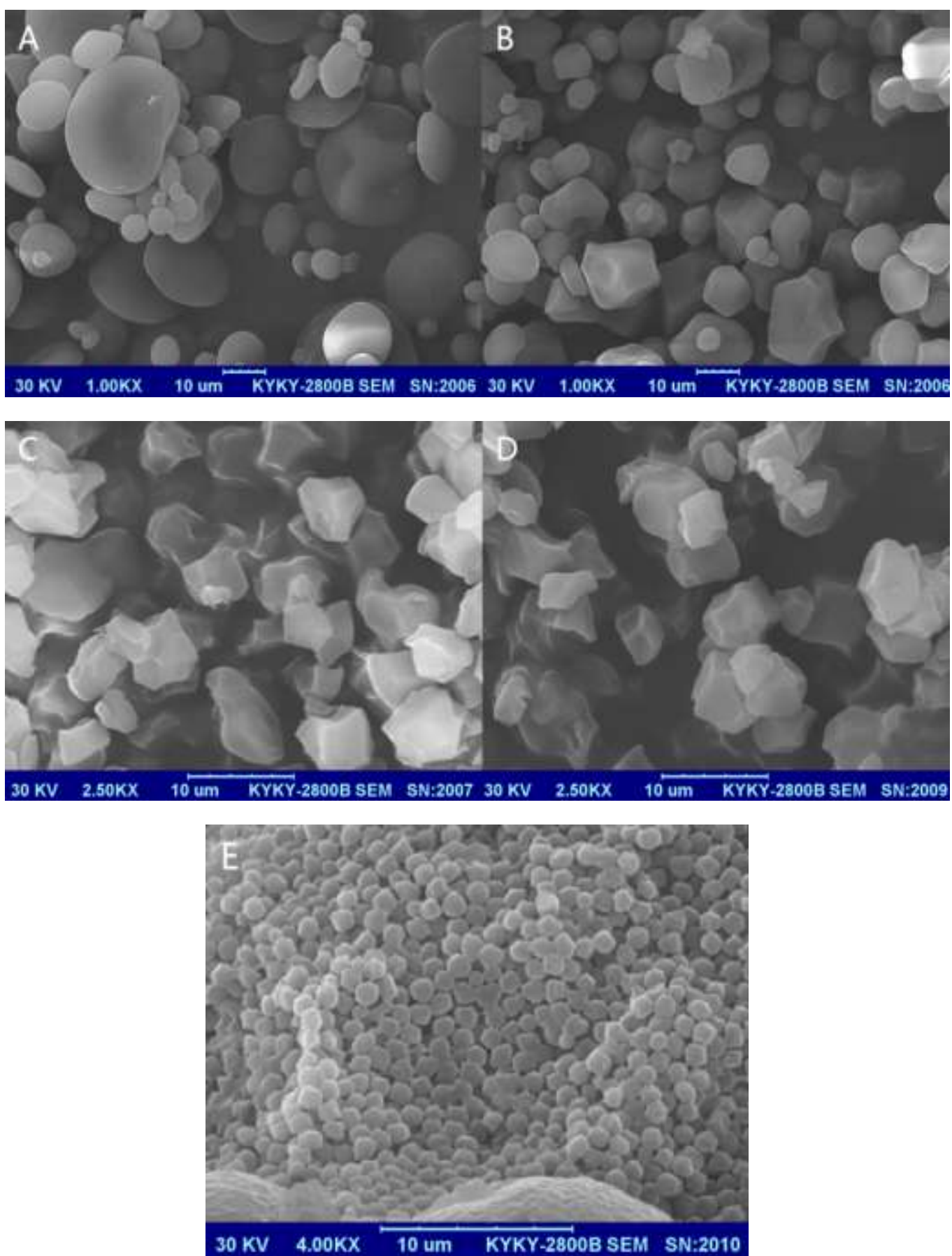

Fig. 1: Scanning electron micrographs of different types of starch granule. A, wheat; B, corn; C, rice; D, waxy rice; E, quinoa 


\section{Freeze-Thaw Stability of Starch}

After freezing and thawing, the wheat starch paste were gelatinous and dense and had a sponge-like structure while the waxy rice and quinoa starch pastes were granular with a loose structure. This state may have been related to the distribution of the branched chains and the chain length of the amylopectin. The molecular structure of starches therefore caused notable differences to the microstructure and the textural properties of the starch gels. After freezing and thawing, the percentage of branched chains (Degree of Polymerization (DP) 18 23) has been shown to be negatively related to the springiness and cohesiveness of the starch gels, while the percentage of medium chains (DP 12 17) was positively related to the springiness of the starch gels (Wang et al., 2013). One possible mechanism for the formation of the double helix structure of oligosaccharides requires at least 10 glucose units rather than short chain molecules, so forming a double helix system depends mainly on the branched molecules of medium length. After freeze-thaw treatment, a large number of these medium length amylopectin molecules were destroyed or freely released. Quinoa amylopectin has been reported to contain extremely high numbers of longest chain $(13.0 \%)$ and short chains, with a few long chains and, as a waxy amylopectin, had a unique distribution of chain lengths (Tang et al., 2002).

\section{Scanning Electron Microscopy}

The granular structure of starches from different cereals showed a significant variation in size and shape as shown by scanning electron microcopy (Fig. 1). Studies have shown that the shape and size of starch granules can affect the efficiency of amylase during enzymatic hydrolysis (Tester et al., 2004). Particle size has been shown to have a greater influence on the activity of starch granules hydrolyzed by $\alpha$-amylase than starch concentration, (Kong et al., 2003). Particle size distribution and particle size are important factors affecting the digestion of cereal starch granules (Al-Rabadi et al., 2009). The particle shape mainly affects the specific surface area and the enzymatic hydrolysis: The greater the specific surface area, the easier the hydrolysis (Tester et al., 2006).

The granular structure of starches from different cereals showed a significant variation in size and shape as shown by scanning electron microcopy.

The quinoa starch granules were polygonal, similar in shape to corn, millet, rice and waxy rice starch granules (Table 4). They were also smaller than all the other starch granules studied and of a uniform size while the size of the other starch granules varied greatly.

\section{Discussion}

Amylose influences certain thermal characteristics of starch such as gelatinization and pasting (Jane et al., 1999;
Sasaki et al., 2000). Amylopectin provides an ordered structure and crystallinity while amylose is present in a more amorphous form. The more highly ordered structures conveyed by amylopectin require more energy to disrupt (Yuryev et al., 2004). Amylopectin is more easily affected by amylase than amylose because it contains more branched chains (Oates, 1997). The ratio of amylose to amylopectin also influences starch retrogradation, a major issue in the staling of food products (Hug-Iten et al., 2003). Overall, the amylose and amylopectin contents and the ratio of amylose to amylopectin significantly affect the functionality and nutritional properties of quinoa starch. Its retrogradation properties, susceptibility to enzymes, gelatinization and pasting properties are also connected to these factors.

A degree of retrogradation can increase the hardness of the starch gel and so contribute to the formation of toughness. However, highly gelatinized and non-aging products are preferred because gelatinized food is easier to digest and be absorbed by the human body (Santa Cruz and Sfara, 2018). Highly gelatinized foods also tend to be soft and palatable in terms of taste and quality, so are popular with consumers. In addition, for manufacturing convenience foods, such properties of starch allow the food to be quickly gelatinized after little processing and easily digested by amylase, so can cater to a fast-paced lifestyle. Thus the quality of stored food can be maintained for a certain period of time and so extend its shelf life. Therefore, quinoa starch would be the most suitable of the starches studied for producing convenience foods and so promote the application of quinoa products.

According to the data, the specific surface area of quinoa starch granules was the largest, so would have had the greatest influence on the efficiency of enzymatic hydrolysis compared with the other starches. Quinoa starch has been shown to exhibit a high enzyme susceptibility.

\section{Conclusion}

The amylose and amylopectin contents, freeze-thaw stability, viscosity characteristics and SEM images of wheat, corn, waxy rice and rice and quinoa starch were compared and analyzed. The high proportion of amylopectin in quinoa starch and the smallest specific surface area of the quinoa starch granule can be related to its high susceptibility to enzymes. For quinoa, the ratio of amylose to amylopectin was high, which have affected the pasting properties and anti-aging properties of its starch. The anti-aging properties of quinoa starch can also be related to its poor freeze-thaw stability. Its loose state can be related to the distribution of the quinoa amylopectin and the length of its branched chains. Quinoa starch showed good thermal stability and the best aging resistance of the starches, because of more branching 
chains allowing it to develop slowly from disorder to order. This article only studies the structure and properties of quinoa starch, but has not studied the physical and chemical properties of quinoa and its commercial application has certain limitations. In the future, we should focus on studying the structure and characteristics of quinoa and combine the results to determine the direction of quinoa commercialization and deep processing.

\section{Acknowledgement}

This study was financially supported by The National Key Research and Development Program of China (2016YFF0202300). We thank Philip Creed, PhD, from Liwen Bianji, Edanz Group China (www.liwenbianji.cn/ac), for editing the English text of a draft of this manuscript.

\section{Author's Contributions}

Menyue Jiao: Participated in all experiments.

Yumei Gao: Collect raw materials.

Wei Zhang: Partial data analysis.

Yiling Tian: Designed the research plan and organized the study.

\section{Conflict of Interest and Funding}

We declare that none of the work contained in this manuscript is published in any language or currently under consideration at any other journal and there are no conflicts of interest to declare. All authors have contributed to, read and approved this submitted manuscript in its current form.

\section{References}

Ahamed, N. T., Singhal, R. S., Kulkarni, P. R., \& Pal, M. (1996). Physicochemical and functional properties of Chenopodium quinoa starch. Carbohydrate polymers, 31(1-2), 99-103.

Al-Rabadi, G. J., Gilbert, R. G., \& Gidley, M. J. (2009). Effect of particle size on kinetics of starch digestion in milled barley and sorghum grains by porcine alphaamylase. Journal of Cereal Science, 50(2), 198-204.

Bertoft, E., Piyachomkwan, K., Chatakanonda, P., \& Sriroth, K. (2008). Internal unit chain composition in amylopectins. Carbohydrate Polymers, 74(3), 527-543.

Hug-Iten, S., Escher, F., \& Conde-Petit, B. (2003). Staling of bread: Role of amylose and amylopectin and influence of starch-degrading enzymes. Cereal Chemistry, 80(6), 654-661.

Jacobs, H., Eerlingen, R. C., Clauwaert, W., \& Delcour, J. A. (1995). Influence of annealing on the pasting properties of starches from varying botanical sources. Cereal chemistry (USA).
Jane, J. L., Chen, Y. Y., Lee, L. F., McPherson, A. E., Wong, K. S., Radosavljevic, M., \& Kasemsuwan, T. (1999). Effects of amylopectin branch chain length and amylose content on the gelatinization and pasting properties of starch. Cereal chemistry, 76(5), 629-637.

Kaufman, R. C., Wilson, J. D., Bean, S. R., Herald, T. J., \& Shi, Y. C. (2015). Development of a 96-well plate iodine binding assay for amylose content determination. Carbohydrate polymers, 115, 444-447.

Kong, B. W., Kim, J. I., Kim, M. J., \& Kim, J. C. (2003). Porcine pancreatic $\alpha$-amylase hydrolysis of native starch granules as a function of granule surface area. Biotechnology progress, 19(4), 1162-1166.

Kusumah, S. H., Andoyo, R., \& Rialita, T. (2020). Isolation and Characterization of Red Bean and Green Bean Protein using the Extraction Method and Isoelectric pH. SciMedicine Journal, 2(2), 77-85.

Linsberger-Martin, G., Lukasch, B., \& Berghofer, E. (2012). Effects of high hydrostatic pressure on the RS content of amaranth, quinoa and wheat starch. Starch-Stärke, 64(2), 157-165.

Liu, J. T., Zhao, K., Liu, N., Yang, C. H., \& Chen, F. L. (2012). Research advances in starch esters [J]. Science and Technology of Food Industry, 20.

Navruz-Varli, S., \& Sanlier, N. (2016). Nutritional and health benefits of quinoa (Chenopodium quinoa Willd.). Journal of Cereal Science, 69, 371-376.

Oates, C. G. (1997). Towards an understanding of starch granule structure and hydrolysis. Trends in Food Science \& Technology, 8(11), 375-382.

Sae-Kang, V., \& Suphantharika, M. (2006). Influence of $\mathrm{pH}$ and xanthan gum addition on freeze-thaw stability of tapioca starch pastes. Carbohydrate Polymers, 65(3), 371-380.

Santa Cruz, S. M, \& Sfara, V. (2018). Chemical signals are involved in the detection and preference of food sources in blattella germanica. Emerging Science Journal 2(5):261.

Sasaki, T., Yasui, T., \& Matsuki, J. (2000). Effect of amylose content on gelatinization, retrogradation and pasting properties of starches from waxy and non-waxy wheat and their F1 seeds. Cereal Chemistry, 77, 611-616.

Steffolani, M. E., León, A. E., \& Pérez, G. T. (2013). Study of the physicochemical and functional characterization of quinoa and kañiwa starches. Starch-Stärke, 65(11-12), 976-983.

Tang, H., Watanabe, K., \& Mitsunaga, T. (2002). Characterization of storage starches from quinoa, barley and adzuki seeds. Carbohydrate Polymers, 49(1), 13-22. 
Teng, L. Y., Chin, N. L., \& Yusof, Y. A. (2013). Rheological and textural studies of fresh and freeze-thawed native sago starch-sugar gels. II. Comparisons with other starch sources and reheating effects. Food Hydrocolloids, 31(2), 156-165.

Tester, R. F., Karkalas, J., \& Qi, X. (2004). Starchcomposition, fine structure and architecture. Journal of cereal science, 39(2), 151-165.

Tester, R. F., Qi, X., \& Karkalas, J. (2006). Hydrolysis of native starches with amylases. Animal feed science and technology, 130(1-2), 39-54.

Wang, L., Xie, B., Xiong, G., Wu, W., Wang, J., Qiao, Y., \& Liao, L. (2013). The effect of freeze-thaw cycles on microstructure and physicochemical properties of four starch gels. Food Hydrocolloids, 31(1), 61-67.
Wang, S. Q., WANG, L. L., FAN, W. H., CAO, H., \& CAO, B. S. (2011). Morphological analysis of common edible starch granules by scanning electron microscopy. Food Science, 32(15), 74-79.

Watanabe, K., Peng, N. L., Tang, H., \& Mitsunaga, T. (2007). Molecular structural characteristics of quinoa starch. Food science and technology research, 13(1), 73-78.

Yan, Z., Jun-Chao, L. I., Zhong-Dong, L., Tao, C., \& Peng, L. (2010). Study on properties and application of waxy wheat starch. Food and Technology.

Yuryev, V. P., Krivandin, A. V., Kiseleva, V. I., Wasserman, L. A., Genkina, N. K., Fornal, J., ... \& Schiraldi, A. (2004). Structural parameters of amylopectin clusters and semi-crystalline growth rings in wheat starches with different amylose content. Carbohydrate Research, 339(16), 2683-2691. 